\title{
El Curriculum nulo de la formación de Profesores de Historia
}

Prof. Paula Inés Sofia*

El presente trabajo surge de una profundización de algunos aspectos de una investigación más amplia, llevada a cabo por un grupo de investigadores de la Universidad de Buenos Aires que estudiamos la Formación de Profesores de Historia en el período 1976-94, y que cuenta con financiamiento de UBACYT.

En dicho proyecto investigamos la formación inicial a través del análisis de los planes de estudio y programas como organizadores previos, los formadores de formadores y los estudiantes como grupo social, en diferentes instituciones de nivel universitario y terciario no universitario.

Es notorio que desde la sanción de la Ley Federal de Educación y la percepción más o menos generalizada de crisis educativa (ya sea por insatisfacción con los resultados de la escolarización o por los procesos de reforma política) la cuestión de la formación docente ha salido a escena y se ha convertido en objeto de estudio de diversas investigaciones; fundamentalmente porque se considera al profesor y su transformación como pieza clave en cualquier intento de mejora de la calidad educativa. Si bien los estudios sobre el profesorado tuvieron su eclosión en el mundo anglosajón en los años '70 y su correlato español a partir de los '80, en nuestro país su desarrollo es aún incipiente y son muy pocos, además, los trabajos que analizan en particular la formación de profesores de Historia.

En esta comunicación nos centraremos en algunos aspectos que llamaron nuestra atención al analizar la dimensión epistemológica de la formación, a partir de la lectura de las encuestas efectuadas a los alumnos-futuros profesores durante el año 1997 y la contrastación con el estudio de los planes y programas de las carreras.

Si bien las conclusiones a las que arribaremos aquí pueden hacerse extensibles, en

*Universidad Nacional de Buenos Aires 
muchos de sus aspectos, a todos los institutos estudiados, en el presente trabajo enfocaremos especialmente el caso del Profesorado de Historia de la Universidad de Buenos Aires, ya que es el que hemos analizado personalmente.

La investigación de los estudiantes del profesorado como grupo social se ha efectuado desde una doble dimensión —-material y simbólica—. Por un lado, en el nivel material y desde un enfoque sociológico en formación docente, hemos estudiado la composición social de origen, edad, género, biografía escolar, nivel educativo de los padres y ejercicio de actividad laboral simultánea con sus estudios.

El estudio desde la dimensión simbólica se inscribe en la orientación del pensamiento del profesor en formación docente, la cual considera a éste como un profesional que toma decisiones. ${ }^{(1)}$ En este nivel hemos analizado las motivaciones para la elección de la carrera, la evaluación que hacen de la misma, las representaciones sobre la profesión elegida y su concepción de la Historia y su enseñanza en el nivel medio. Metodológicamente hemos utilizado encuestas semiestructuradas para recoger la información, en una muestra constituida por alumnos del primero y del último año de la carrera.

Si bien los resultados más generales de esta etapa de la investigación fueron presentados en otro trabajo, ${ }^{(2)}$ queremos profundizar aquí algunos aspectos claves que se derivan del análisis de la dimensión simbólica (puntualmente, en las concepciones epistemológicas y la evaluación de la carrera); los cuales al aparecer como recurrencias en otros niveles del proyecto, tales como el análisis cualitativo del plan de estudios de la carrera y de los programas, nos permiten adelantar la hipótesis de la existencia de un curriculum nulo en la formación.

La conceptualización teórica sobre los diferentes tipos de currícula nos permite considerar no sólo lo que las instituciones enseñan a través de su curriculum explícito e, implícitamente, a través del curriculum oculto, sino también lo que estas instituciones no enseñan.

Si adscribimos al argumento que plantea que "la ignorancia no es simplemente un vacío neutral", ${ }^{(3)}$ debemos aceptar que muchas veces lo que no se enseña es tan importante como lo que se enseña; y que la ausencia de conjuntos de saberes (ya sea en la dimensión de los contenidos o en la de procesos intelectuales) en la formación inicial de un profesor de Historia tendrá consecuencias, seguramente, en la estructuración de su pensamiento como docente, en la incapacidad para utilizar ciertos procesos, en la imposibilidad de considerar determinadas perspectivas y en las opciones que sea capaz de ofrecer a sus alumnos.

Las ausencias que analizaremos aquí, ligadas al conocimiento epistemológico de nuestra disciplina y a la metodología de investigación del historiador, y que incluimos en el curriculum nulo de la formación, posiblemente no sean las únicas. Pero en el caso de la enseñanza de la Historia son decisivas. 
Hablar de cuestiones epistemológicas en el conocimiento histórico significa siempre reflexionar sobre el ser humano y sus acciones como individuo social, lo cual confiere a la enseñanza de la Historia un potencial intelectual y también educativo enorme. Pero ello es así a condición de que esa reflexión se explicite. ${ }^{(4)}$

Una de las cuestiones que surgen de las encuestas realizadas a los futuros profesores que más ha llamado nuestra atención es la pobreza — cuando no desconocimiento absolutosobre aspectos de teoría o epistemología histórica verificada en las respuestas. Asimismo, se advierten serias dificultades para precisar algunos objetivos generales de la enseñanza de la asignatura; lo cual debería interpretarse como lógico correlato de dicha pobreza teórica, ya que sabemos que las ideas sobre el para qué de la enseñanza de la historia dependen, en gran medida, de la concepción epistemológica de la misma que se sustente.

Ante la pregunta — de respuesta absolutamente abierta — sobre cuál sería para ellos la importancia de la enseñanza de la Historia en la escuela secundaria, más del 30\% de los alumnos de $1^{\circ}$ año directamente no responde o lo que expresa resulta ser tan confuso, incoherente o impreciso, que se deduce que no puede definir la importancia de la Historia o una concepción de la misma. Entre los alumnos de $1^{\circ}$ año que sí respondieron a esta pregunta, la mayoría de las respuestas se asocia — aunque de manera muy confusa - a una concepción renovada o, por lo menos, crítica de la historia factual, vinculada a formar un pensamiento crítico o a conocer/comprender la realidad. En un porcentaje mucho menor (menos del $10 \%)$ aparece mencionado el valor de la historia como "maestra de vida" asociada a una concepción clásica. No se registran prácticamente menciones vinculadas a una concepción tradicional de la historia o ligadas a recuperar valores del pasado relacionados con la identidad nacional; tampoco menciones explícitas a una actitud comprometida con la realidad social ligadas al conocimiento del pasado para intervenir en el presente.

De todas formas, estos datos pueden no ser llamativos si consideramos que la mayoría de los alumnos encuestados de $1^{\circ}$ año manifestó haber ingresado a la carrera ese mismo año - es decir, unos meses antes de realizarse la encuesta- Así, es factible suponer que sus ideas sobre la historia son a priori o previas al ingreso a la carrera, probablemente las que traen de su paso por la escuela media.

En cambio, son elocuentes al respecto las respuestas de los alumnos del último año. En primer lugar, porque en ellas se mantiene la misma proporción (30\%) de alumnos que no puede responder o expresa ideas tan confusas, incoherentes o imprecisas que se deduce su imposibilidad de definir alguna concepción de la disciplina. En segundo lugar, porque del $70 \%$ restante se asocia a concepciones muy similares a las de los alumnos de $1^{\circ}$ año y se 
mantiene la pobreza de la mayoría de las respuestas, con serias dificultades para definir (o tan siquiera mencionar) conceptos o ideas básicas de la epistemología histórica. De este modo, las respuestas reflejan un enorme desconocimiento sobre las discusiones teórico-epistemológicas en el seno de la disciplina en las últimas décadas y tampoco dan cuenta de un manejo razonable de las posiciones de las diferentes corrientes historiográficas del último siglo.

Del análisis de las respuestas de estos alumnos próximos a concluir su Profesorado, y considerando su prácticamente inexistente diferenciación respecto del tipo de respuestas de los recién ingresados, es posible inferir el bajo impacto de la carrera en la formación teórica y epistemológica de los futuros profesores. Esta hipótesis inicial se verá reforzada con el análisis de los planes de estudio y los programas de las materias y seminarios que dan cuenta de recurrencias en este sentido, según veremos más adelante.

Otro de los aspectos que incluimos en el curriculum nulo de la formación de profesores es el referido a la metodología de investigación histórica. También partimos, en este caso, de las encuestas a los futuros graduados. Entre los aspectos negativos de la carrera (de respuesta abierta y múltiple) uno de los manifestados por la mayoría de los estudiantes del último año es la falta de formación en metodología de investigación —aspecto que nosotros consideramos íntimamente relacionado con la deficiente formación teórica.

A su vez, no casualmente, otro de los puntos más criticados por los futuros formadores es el régimen de evaluación en el cual predominan los exámenes finales y parciales tradicionales. El predominio de este tipo de evaluación tradicional está generalmente ligado al "control de lectura”, que no exige ningún tipo de elaboración de conocimiento histórico por parte de los futuros profesores y se limita a verificar sólo su conocimiento sobre los resultados de la investigación histórica, realizada por otros. Consideramos que esto no es casual porque cuando la formación no incluye adiestramiento metodológico y ejercitación paulatina en el saber-hacer del historiador, los métodos de evaluación tradicionales y reproductivos son casi un correlato previsible.

Como veremos ahora, nuestra interpretación respecto de las falencias de la carrera en las dimensiones teórico-epistemológica y metodológica y la inclusión de estas dimensiones en el curriculum nulo de la formación inicial se ve reforzada con el análisis de ciertos aspectos de los planes de estudio y programas de las materias y seminarios.

En este caso, sólo tomaremos las cuestiones relacionadas con este tema del plan de estudios vigente y de los programas del período 1985-95. (5) El plan de estudios actual de la carrera de Historia entra en vigencia, con el regreso de la democracia política, en el año 1985 (aprobado por Resolución del Consejo Superior Provisorio No 468/85). Para obtener el título de Profesor de Enseñanza Media y Superior en Historia se exige la aprobación de 32 
cursos cuatrimestrales —incluido el Ciclo Básico Común- de los cuales el 63\% corresponde al saber disciplinar, el 31\% a la formación general y el 6\% al saber pedagógico.

Esta mínima proporción destinada al saber pedagógico inscribe al Profesorado en la denominada "tradición académica". En el estudio de la formación docente se han configurado tradiciones ${ }^{(6)}$ encarnadas en los sujetos y en las instituciones que muchas veces las reformas acaban reforzando; entendemos estas tradiciones como configuraciones de pensamiento y acción que, construidas históricamente, se mantienen a lo largo del tiempo, en cuanto están institucionalizadas, incorporadas a las prácticas y a la conciencia de los sujetos.

Una de estas tradiciones es la académica, que plantea que los docentes deben conocer sólidamente la materia que enseñan mientras que la formación pedagógica es secundaria. En una situación extrema, cuestionan los cursos de formación pedagógica, considerados triviales, sin rigor científico y plantean que tales conocimientos pueden adquirirse en la práctica, dado que cualquier persona con buena formación y "sentido común" conseguiría orientar la enseñanza; es decir, hay una identificación casi automática entre saber la asignatura y saber enseñar.

En la Argentina, el mayor impacto de esta tradición se ha producido en la preparación de docentes para la enseñanza secundaria, tanto en institutos universitarios como terciarios. De esta manera, el profesor formado en estas instituciones suele estar mucho más identificado con su disciplina que con la enseñanza; así, los profesores de Historia se perciben a sí mismos mucho más como historiadores que como profesores. ${ }^{(7)}$ Sin embargo, la formación parece ser poco profesionalizadora tanto en uno como en otro sentido. Al escaso espacio destinado a la formación y las prácticas pedagógicas se suma la escasa preparación profesional para ser historiador.

En este último sentido, el plan de estudios plantea como objetivo "una formación sistemática orientada hacia la tarea de investigación, es decir, que enfatizará en el graduado aquellas aptitudes que lo individualizarán como transmisor productor de conocimiento". ${ }^{(8)}$ A pesar de esta declaración, el análisis de los programas de las materias y seminarios del período no parece dar cuenta de dicha "formación sistemática". Los programas, si bien muy renovados en cuanto a contenidos y bibliografía y marcando importantes rupturas en sus ejes respecto del período dictatorial anterior, carecen en general de tópicos ligados al entrenamiento del oficio, como podría ser el análisis de fuentes en un sentido no meramente manipulativo o ejemplificativo, la elaboración de pequeñas investigaciones o la inclusión de métodos de evaluación en los que no predomine lo reproductivo. La formación en investigación, entonces, parece no ser sistemática y queda relegada a los seminarios a cursar hacia el final de la carrera. Estos seminarios, según el mencionado plan de estudios, se dividían entre "semina- 
rios de metodología de la investigación histórica" y "seminarios que desarrollen un tema, problemática o aspecto de la orientación elegida"; del análisis de los programas de los seminarios del período se deduce que, si bien prácticamente ninguno hace explícita su pertenencia a uno u otro tipo, la mayoría está destinada a la profundización bibliográfica de un tema y, en más de la mitad, no figuran fuentes en su bibliografía.

Estas deficiencias en la formación en metodología de investigación se transforman llanamente en parte del curriculum nulo con la reforma al plan de estudios realizada en el año 1992 que aprueba, para el profesorado, la reducción del número de seminarios de cuatro a dos "pudiendo ser uno temático y uno de investigación o los dos temáticos". (9) Esta reforma, finalmente, viene a legitimar una situación de hecho, excluyendo del curriculum explícito del profesorado los seminarios de metodología de investigación y proponiendo seminarios temáticos cuyo "resultado esperado es la redacción del análisis crítico de un corpus bibliográfico y, eventualmente, de un estado de la cuestión"; (10) lo cual, contrariamente a lo expresado entre los objetivos del plan de estudios, refuerza el rol de transmisor-reproductor del futuro profesor.

En el análisis de los planes y programas aparecen, también como recurrencias, las falencias en la formación teórica y epistemológica, confirmando los resultados de las encuestas abiertas. La pobreza teórica de los estudiantes tiene parte de su correlato en el plan de estudios, en el cual no figuran materias de filosofía o epistemología de la Historia, ni tan siquiera una más general epistemología de las ciencias sociales. La asignatura cuatrimestral Teoría e Historia de la Historiografía parece ser la única que, solitariamente, debe cumplir la compleja misión de formar teóricamente a los futuros formadores; el análisis de sus programas en el período da cuenta de un eje preferentemente centrado en la historia de la historiografía más reciente (el recorte parece inevitable en el breve lapso de un cuatrimestre), quedando los problemas más generales de teoría para mejor oportunidad. En los programas del resto de las materias, están generalmente ausentes las precisiones o explicitaciones teóricas, epistemológicas o historiográficas; si bien en algunos figuran, por ejemplo en la bibliografía, autores con posiciones divergentes sobre un mismo tema, estas diferencias historiográficas nunca aparecen explícitamente cuando el tema se incluye entre los contenidos. También son muy poco frecuentes las explicitaciones sobre las concepciones teóricas que respaldan la elección de los ejes o periodizaciones que articulan los contenidos.

De lo anterior se desprende la reafirmación de la existencia de un curriculum nulo en la formación de profesores de Historia conformado, en principio, por el conocimiento teórico-epistemológico de la disciplina y por la metodología o el saber-hacer del historiador. Estas ausencias definirán, seguramente, importantes consecuencias en el pensamiento de los 
futuros docentes y en la configuración de sus transposiciones didácticas que definirán el saber enseñado. Sabemos que las concepciones, teorías y creencias explícitas e implícitas de los profesores son claros condicionantes de la práctica al limitar sus construcciones metodológicas.

Cuando hablamos de construcción metodológica nos referimos ${ }^{(11)}$ al hecho de que en las prácticas de la enseñanza el docente, desde su singularidad, construye conocimiento metodológico al resignificar la articulación entre los contenidos disciplinares y los procesos cognitivos. Esta articulación dialéctica entre forma y contenido se da en situaciones concretas y contextuadas y varía según el estilo y formación del docente, fuertemente impregnados de adscripciones teóricas que influyen en las decisiones sobre el para qué enseñar, qué contenidos enseñar o lo que es o no negociable, ante la inmediatez en que se desarrollan las prácticas. El estilo del docente no sólo enmarca su construcción metodológica sino que, al mismo tiempo, se resignifica como contenido de la clase reflexiva.

La buena enseñanza ${ }^{(12)}$ reflexiva remite a dimensiones epistemológicas y éticas. Decimos éticas y no morales porque consideramos a la moral como una categoría dada para una sociedad determinada en espacio y tiempo; la ética, en cambio, se constituye como un análisis crítico de la moral, lo cual implica la toma de distancia necesaria para develarla y ubicarla en su contexto y sus condiciones históricas de producción.

Desde su dimensión epistemológica, la buena enseñanza de la Historia nos impone la reflexión sobre el para qué como una decisión ética más; pero, al mismo tiempo, las propias estructuras sustantiva y sintáctica de la disciplina nos permiten proporcionar al estudiante los medios para estructurar su propia reflexión crítica sobre la moral históricamente situada. La reflexión epistemológica y metodológica en Historia nos permite develar lo oculto; hacer explícito lo implícito; rastrear los orígenes de lo que se creía eterno, dado, natural; trazar genealogías de lo supuestamente universal. Brindar las herramientas para comprender empáticamente el mundo también es desestructurar el poder-saber ${ }^{(13)}$ en el aula. La Historia — por la propia naturaleza de su objeto— está en mejores condiciones de proporcionar esas herramientas que muchas otras disciplinas, tanto desde sus resultados como desde su estructura sintáctica.

Sin embargo, la clase reflexiva y crítica así planteada presenta como condición necesaria la existencia de un docente formado en la reflexión y la crítica. El pensamiento del profesor influirá implícitamente en la implementación curricular; y el curriculum nulo de su formación se transformará en curriculum nulo de su transposición, con la consecuencia que: 
...las opciones que no se ofrecen a los estudiantes, las perspectivas que quizá ellos nunca conozcan y mucho menos aprendan a usar, los conceptos y habilidades que no son parte de su repertorio intelectual. Seguramente, en las deliberaciones que constituyen el curso del vivir su ausencia tendrá importantes consecuencias en la clase de vida que los estudiantes elijan llevar. (14)

\section{Notas}

${ }^{1}$ Barquín, J.: "La investigación sobre el Profesorado. Estado de la cuestión en España" en Revista de Educación, № 306, Madrid, enero-abril, 1995.

${ }^{2}$ Sofía, P. I.: Formación de profesores de Historia: el perfil de los estudiantes de la UBA, Ponencia presentada en las VII Jornadas Interescuelas/departamentos de Historia, Universidad Nacional del Comahue, Neuquén, setiembre de 1999.

${ }^{3}$ Eisner, E.: The educational imagination. On the Design and Evaluation of School Programs, New York, Mac Millan Publishing, 1985.

${ }^{4}$ Maestro González, P.: “Epistemología histórica y enseñanza” en Ruiz Torres, P.: $L a$ Historiografia, Ayer, Madrid, Marcial Pons, 1993.

${ }^{5}$ Para un análisis comparativo de los planes y programas de los períodos 1976-84 y 1984-94 ver Sofía, P. I.: Rupturas y continuidades en la formación de profesores de Historia, Ponencia presentada en la Jornada "Los que enseñamos Historia”, Universidad de Morón, 1996.

${ }^{6}$ Davini, M. C.: La formación docente en cuestión: política y pedagogía, Buenos Aires, Paidós, 1995.

${ }^{7}$ Torres Bravo, P. A.: "Concepciones, creencias, teorías implícitas y formación del profesorado en Historia” en Clío \& Asociados - La Historia enseñada, No 3, Santa Fe, 1998.

${ }^{8}$ Los subrayados son nuestros.

${ }^{9}$ Resolución de Consejo Directivo No 3784/92.

${ }^{10}$ Resolución de Consejo Directivo Na 3776/92.

${ }^{11}$ Edelstein, G. y Coria, A.: Imágenes e imaginación. Iniciación a la docencia, Buenos Aires, Kapeluz, 1995.

${ }^{12}$ Fenstermacher, G.: "Tres aspectos de la filosofía de la investigación sobre la enseñanza" en Wittrock, M.: La investigación de la enseñanza. Enfoques, teorías y métodos I, México, Paidós, 1989.

${ }^{13}$ Souto, M.: Hacia una didáctica de lo grupal, Buenos Aires, Miño y Dávila, 1993.

${ }^{14}$ Eisner, E.: op. cit. 